\title{
IMPROVING THE POPULARITY OF EDUCATIONAL GAMES USING OPTIMAL GAME DEVELOPMENT
}

\author{
Johann Allers, Gunther R. Drevin and Trudie Benade \\ North-West University, Potchefstroom, South Africa
}

\begin{abstract}
Educational games are powerful tools that can be utilized to help people learn while having fun, but it is one of the least played genres of games to date. This paper addresses the problem of increasing the popularity of educational games by identifying optimal game elements and characteristics and implementing it in an educational game. In other words, the goal was to create a fun-to-play and educational game. A literature survey was done to identify these elements with the goal to create a game which enables a user to play the popular educational game, Scrabble. A desktop application that implemented all of these elements, identified in the study, was created and it allows the user to play Scrabble against artificial intelligence opponents.
\end{abstract}

\section{KEYWORDS}

Educational Games, Optimal Game Development, Scrabble

\section{INTRODUCTION}

Educational games are powerful tools that can be utilized to help people learn while having fun, but it is one of the least played genres of games to date. This paper addresses the problem of increasing the popularity of educational games by identifying optimal game elements and characteristics and implementing it into an educational game.

\subsection{A Brief History of Game-Based Learning}

The computer-based game Pac-Man is one of the most iconic games to date and it has had a big influence on computer games as we know it today even though we do not realise it. One example of the influence that Pac-Man has had is the advancement and creation of educational computer games (Needleman, 2017).

Although Pac-Man is not an educational game by any means, the success of the game changed educators' view of computer games from believing that it is only a distraction or something that is harmful to children, to seeing the potential of games as a medium for teaching (Zin, 2009).

The first computer based game that can be classified as an educational game, was LOGO Programming (Needleman, 2017). LOGO Programming was released in 1967 by the company named Turtle academy, with the goal to teach users how to successfully write code using the LOGO programming language, developed by Papert and Fuerzeig (Abelson, 1974). The goal of LOGO was to program a small cursor (called a turtle) to move around and draw lines using mathematical concepts. Thus in addition to teaching users how to code in the LOGO programming language, it also taught users mathematical concepts in the form of a game.

In 1971, Oregon Trail was developed by history teachers Rawitsch, Heinemann, and Dillenberger and released by MECC in 1974 and is the educational game that had the biggest impact on the use and creation of educational games (Needleman, 2017). Oregon Trail is a text-based game set in the 1800s, in which the user leads an oxen pulled wagon from Missouri to Oregon. Users had to overcome obstacles that were accurate to that time period, making this game an interactive lesson on American history. Oregon Trail was the first educational game to be used in classrooms as a method of education. 
In 1973 the educational game Lemonade Stand was released. The goal of this business simulation game was to teach users the basics of economics by allowing them to manage a lemonade stand. This game had great success, especially among younger users (Zin, 2009).

Following the success of Oregon Trail and Lemonade Stand, more games where developed that focused on educating the user, or had some hidden method to educate users without them realising. Examples of these games include Where in the world is Carmen Sandiego (focused on logic, reasoning and contained elements of geography), Reader Rabbit (focused on developing reading skills and spelling) and Number Munchers (focused on mathematics).

In the late 80's and early 90's, the number of educational games grew exponentially (Zin, 2009). The educational games also explored more genres, such as action, adventure or simulations. The main demographic target of educational game companies in this era was children and although children were the primary users, this limited the growth of educational games drastically.

In the last couple of years, more major companies started developing educational games targeted at users of all ages (Needleman, 2017). Modern development companies also combined the conventional focus of educational games with creative and problem solving elements, to give a better gaming experience to users.

\subsection{Why Educational Games Fail}

A study done at the Massachusetts Institute of Technology (MIT) showed that educational games are a lot less popular than most other gaming genres (Klopfer, 2009). The short explanation to why the popularity of educational games is so low is that the users require games to be fun and educational while being available on many different platforms (Stutt, 2010). In other words, users demand that a lot of criteria be met before they will classify an educational game as good.

One of the biggest reasons why educational games fail is because of the general public's view of computer games in general and computer games as a tool for learning (Stutt 2010). The educational community perceives games as lacking seriousness and therefore they refuse to use it as a tool for education. Furthermore, the cultural and social structure of education is also difficult to change and it is slow to adapt to new technology, proving to be an even larger obstacle (Klopfer, 2009).

Another barrier that educational games and game developers have to face when creating an educational game is the time and cost of development (Klopfer, 2009). Developing games requires a lot of time, money and other resources. For developers to create games that have an uncertain market strength is risky and often leads to failure and therefore it is uncommon for developers to spend resources on educational games in the current, uncertain market. Unless there is a change in the way in which educational institutions view games and their policies regarding educational games as a tool for education, it is unlikely that the market will have excellent or even good educational games (Stutt, 2010).

In short, educational games are stuck in a negative feedback loop (Klopfer, 2009). There is not a lot of money available in the market for educational games and therefore companies refuse to spend a lot of resources developing educational games. This causes the available educational games to have lower quality, providing less income and also causes a shortage of money in the market of educational games.

Developers are also hesitant to create educational games due to the history of failed educational games.

\subsection{The Effectiveness of Game Based Learning}

In the last couple of years, extensive research has been done at a number of educational institutions to determine whether the use of educational games has an impact on learners and what kind of impact that is. Three examples of such studies are:

\subsubsection{Syracuse University}

A study done at the Syracuse University had two main research questions: (1) Do instructional games augment learning? (2) What is the impact of the challenge and fantasy features, in instructional games, on learning? (Amr, 2012)

For this study, four versions of a game, the Humatan game, were developed. These versions were: A game with only the challenge feature turned on; A game with only the fantasy feature turned on; A game with both the challenge and fantasy features turned on; And a game with challenge and fantasy turned off. 
Two-hundred-and-two students at the Baltimore Country Public school where given a test on human anatomy. After the test, each student was assigned randomly to play one of the versions of the game and then retook the test. Each student was also asked to fill in a survey to detect any patterns based on their Grade Point Average (GPA), Socio-economic Status (SES), game skilfulness, gender and ethnicity.

A statistical analysis of the data gathered showed a distinct improvement for all students that participated in the study. The average of the improvement scores through the group is 3.40 with a standard deviation of the improvement score is 3.67 (Amr, 2012).

\subsubsection{Indiana University of Pennsylvania (IUP)}

A study was done at the Indiana University of Pennsylvania's (IUP) Applied Media and Simulation Games Centre to determine what effect educational computer games had on knowledge acquisition (Almeida, 2012).

A group of sixty-five undergraduate students took part in the study. The students were randomly assigned to two groups, a control group and a treatment group. A quiz was set up so that the students would have little knowledge prior to the experiment, so as to rule out pre-existing knowledge as a variable. The control group was given a script and then asked to complete the quiz. The treatment group was given the same script after which they played a game about the subject and then completed the quiz.

The results of the quiz showed that the treatment group scored significantly higher than the control group. The control group had a mean of $76.8 \%$, against the treatment group's mean of $83.5 \%$. This is a difference of $6.7 \%$ and thus an $8.7 \%$ improvement on the control group. The respective standard deviation of the control group and the treatment group was 0.115 and 0.096 (Almeida, 2012).

\subsubsection{University of Piraeus}

Virvou, Katsionis, and Manos from the University of Piraeus' Department of Informatics did a study on the effect of computer based Intelligent Tutoring Systems (ITS) and virtual reality (VR) based Intelligent Tutoring Systems (Virvou, 2005). The goal of the study was to determine if both computer based and VR based ITSs shows an improvement in marks received on school level. The study also aimed to determine whether VR ITSs are superior to computer based ITSs.

For this study a group of 90 grade four students was used. The group was divided into two sub-groups. The one subgroup used a simple computer based ITS, and the other used a virtual reality version. After the students completed this phase, they were asked to complete a test that was similar to what they would write in school. The results of these tests were collected and compared to their usual results, before they were exposed to the ITSs, based on the number of mistakes made (Virvou, 2005).

The analysis of the study showed that the use of computer based games greatly improves the results that the students achieve in school. The study concluded that this is largely due to the fact that the students are motivated and that they are able to focus better when playing the games.

\subsubsection{Summary}

The results of these three studies show that educational games have a positive effect on the results of students when used as a tool for learning as well as having a positive effect on the morale, motivation, concentration and enjoyment of students. These results are further supported by a study done at the University of Alcalá in which an analysis was done of one-hundred different papers that did studies on the effectiveness of educational games as a tool for learning for different categories (Merino-Campos, 2016). These categories include the improvement of attitude, intellectual skills, knowledge, motor skills and physical properties. The results show that $84 \%$ of papers studies showed an improvement in one or more of the categories.

\section{CHARACTERISTICS OF OPTIMAL GAMES}

To better understand the scope of what an optimal game requires, this section will focus on summarizing different elements that are necessary when creating games and what to keep in mind when developing optimal games.

Two of the most influential people in the history of educational games and educational game development are Malone and Lepper (Mildner, 2015). Both Malone and Lepper's research focuses on one central statement: By enhancing the amount of fun the user is having, their motivation will also increase and therefore users will 
be more engaged when playing the game (Malone, 1987). Malone and Lepper believed that creating a game that is fun is the key to creating a successful educational game (Malone, 1987).

In 1981, Malone defined the four most important characteristics of educational games that focuses on the motivation of the users and is still used today as the foundation of any educational games. These four characteristics are challenge, fantasy, curiosity and choice (Malone, 1981). Charsky (2010) went on to describe two more characteristics, namely rules, and competition and goals. Malone's characteristics were also adapted by Hunicke, Leblanc and Zubek, turning the focus to the aesthetic characteristics of games (Hunicke, 2004). They defined eight characteristics, namely sensation, fantasy, narrative, challenge, fellowship, discovery, expression and submission. Kramer (2015) identified fifteen criteria necessary for creating a good game. These criteria include: originality, freshness and replay-ability, surprise, equal opportunity, no 'Kingmaker effect', no early elimination, reasonable waiting times, creative control, uniformity, quality of components, target groups, tension, learning and mastering a game and complexity and influence

Due to the large number of characteristics, only a few will be discussed in this paper. In the literature the following characteristics have been identified as the most important: Challenge, fantasy, curiosity, choice, rules, and competition and goals.

\subsection{Challenge}

Challenge is one of the original criteria for educational games identified by Malone and it is also acknowledged by most other literature as an important factor in educational games. According to Malone and Lepper (Malone, 1987), the challenges of a game are the tasks and activities of that game. The characteristic of challenge was also described by Mildner (2015), as the problem that will require the user's skills or abilities to overcome. The challenge element is often seen as the main element of games and the challenges of educational games are usually set up in such a way that by completing the challenges, the user gains new skills and a better understanding of the game, mechanics and content. This leads the user to more complex challenges, which also allows the user to gain more advanced skills. Thus challenges can be viewed as the main method of learning in educational games and as challenges are completed, more complex challenges arise, further expanding the user's skills and knowledge.

\subsection{Fantasy}

According to Charsky (2010), nearly every educational game contains some element of fantasy. The main reason that most educational games use this is to attempt to motivate the users and provide them with an exciting gameplay experience. Fantasy can be divided into two subgroups: exogenous or endogenous. Exogenous fantasy is used as a method of rewarding the user for correct behaviour. This is most commonly used as a reward when completing a given task. A common way of applying this method is by rewarding the user with another piece of lore or plot of the game. According to Malone and Lepper (Malone1987), there is a disconnection between the challenge and fantasy of a game. This is the reason why most edutainment games are classified as drill and practice activities disguised as games. Endogenous fantasy uses fantasy as a method to assist in expanding the knowledge of the user, rather than just acting as a reward. There is little to no disconnect between the challenge and the fantasy aspect when using this method.

\subsection{Curiosity}

Malone (1981) describes curiosity as one of the most important features of motivation. The main goal of this characteristic is to stimulate and then satisfy the user's curiosity about the game and certain aspects or knowledge of the game. Studies have shown that people spend more time looking at complex stimuli in a pair of similar images (Malone, 1981). This shows that people are more focused on complexity because it stimulates curiosity. The most common way to achieve this curiosity level is to integrate visual effects and complex problems that the user has to solve. 


\subsection{Choice}

The choice characteristic of games refers to the available options that a user has prior to, and during gameplay (Charsky, 2010). In other words, the choices are the available decisions that a user has to make in order for the game to progress. There are three main choice categories: Expressive, Strategic, and Tactical. An expressive choice usually has little to no effect on gameplay or learning experience, but is focused on improving the user's motivation (Malone, 1981). Expressive choice is typically a method in which users are able to express themselves, also allowing them to relate more to the game. Examples of these choices are picking or creating an avatar, picking a name for the avatar, customizing certain visuals etc. A strategic choice is a choice that has a direct influence on the way the game is played. This is usually done by changing certain game attributes. These decisions focus on allowing the user to be challenged on a level that they desire and for them to be in charge of their game experience (Charsky 2010). The most common examples of strategic choices are being able to choose a difficulty level, selecting a timeframe, number of players etc. The final type of choice is tactical choice. This gives the users the ability to decide how they play the game. This means that the users will be prompted to make a decision, and the game may adapt based on their decision. The most common way that these choices work is by allowing the users to be able to choose an option A, but also allowing them to not choose A and rather select a different option (Charsky, 2010). The most common use of this choice is in-game decisions and usually only affects the story.

\subsection{Rules}

According to Charsky (Charsky2010), rules are the limits on what actions a user may take. Rules are an important factor in educational games, because it gives a sense of reality. This means that the rules in educational games represent rules in real life that users should learn to follow. Rules are also used to define the scope of the game and how to play it properly. This can also describe additional factors, such as adding more players. The rules in educational games are usually set and cannot be broken.

\subsection{Competition and Goals}

The final characteristic identified by Charsky (Charsky2010), is the competition or goals. In contrast to the rules, the goals inform the user of the actions that they are allowed to take. This also clearly defines the conditions for winning the game and how to play the game (Charsky, 2010). An example of the goals is capturing the opponent's king in a game of chess, completing all challenges given etc. The competition aspect of this characteristic includes pitting the user against another player. This player is typically another user or an AI program.

\section{SCRABBLE}

Scrabble is an educational board game that allows players to create words with a limited number of letters. Scrabble is highly strategy based and focuses on increasing the player's vocabulary and spelling abilities. 


\subsection{Gameplay and Rules}

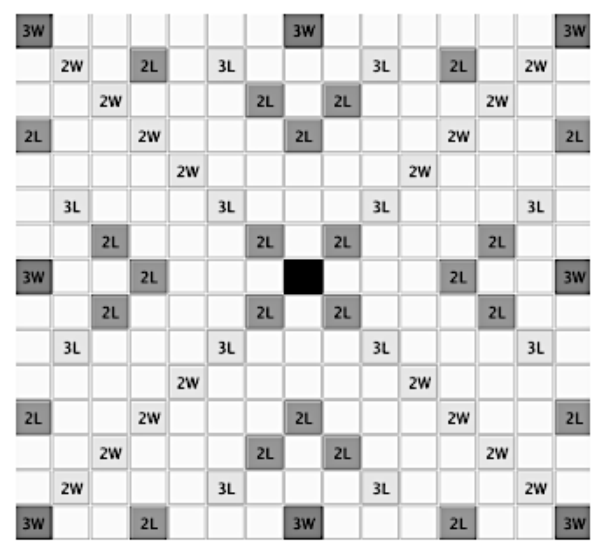

Figure 1. Layout of standard Scrabble board

\subsubsection{Components}

The game of Scrabble is played on a board, marked with a grid, and a set of tiles. The main components of the game are:

- A 15x15 board with certain highlighted squares (Figure 1). These squares are bonus squares and will be discussed in the rules.

- Tiles marked with letters and numbers. The letters represent the letters used to spell a word and the number represents the score of playing that letter.

\subsubsection{Objective}

When starting the game, each player has to randomly select seven tiles. The first player has to spell a word on the board by placing tiles horizontally or diagonally on the board (one tile per block). The first word has to cross the centre of the board. After the word is created, players take turns adding words to the board, with the condition that each word has to cross with or use a letter or letters from words already on the board and that after adding a word, all existing words still make sense. After a word is played, the score of the word is calculated and added to the player's score.

\subsubsection{Turns}

Player have two options when it is their turn: First, they can lay out a word on the board and pick up the number of tiles that they played and secondly, they can pass their turn, receiving no points.

\subsubsection{Bonus Squares}

There are four types of bonus squares: Double letter, triple letter, double word and triple word. These bonuses are added to the score during the calculation phase only if the player has placed a tile on that position. The following list explains certain rules when bonus squares are used:

- The double and triple letter multiplies the value of the letter that is placed on the square with two and three respectively. The double and triple word does the same thing, but it multiplies the score of the entire word instead of just the single letter.

- Each bonus square can only be used once, when the tile is first placed on it.

- Letter bonuses are calculated before word bonuses.

- An additional 50 points are added if a player uses all the tiles they have in one move.

\subsubsection{Ending the Game}

There are three possible ways of ending the game. First is when players reach a predetermined goal, second is when a player's rack is empty and there are no more available tiles and lastly, when all players agree that they cannot make a move. The condition for winning is having the highest score at the end of the game. 


\subsection{Scrabble Strategies}

The following is a short list and explanation of different strategies that can be used when playing Scrabble.

Play difficult letters as soon as possible (Connolly, 2012). Despite the fact that difficult letters have higher points, they still might not be a part of the word that will give the player the highest score. In these situations, it is important to remember that these letters are difficult to place. Waiting for a better chance to play the letter, might lead to the opportunity slipping past, and the letter becomes a burden on the rack, taking up the space of a letter that might be useful.

Maintain a balance on the rack (Connolly, 2012). When spelling words, we use a lot more consonants than vowels, but vowels make part of every word. This makes it easier to play vowels when playing scrabble. By quickly playing all of the vowels that a player has, they may limit themselves in next rounds. By keeping a balance between vowels and consonants, the player will have a broader selection when playing a word.

Play bonus squares (Connolly, 2012). Not only does playing a bonus square increase the points awarded for a word significantly, but it also prevents other players from using that bonus square. By focusing on bonus squares, players can increase their own score while limiting the scores of opponents.

Focus on word extensions by using prefixes or suffixes (Connolly, 2012). By extending a word, the player is able to gain a lot of points for existing letters. This also allows the player to gain points without using up most characters on the rack.

The final strategy is to focus on expanding one's vocabulary (Connolly, 2012). By expanding their vocabulary, players are able to identify more words and thus have more options of moves when playing Scrabble.

\section{DESCRIPTION OF IMPLEMENTATION}

To best describe the artefact, it will be discussed by referring to the core elements, identified in literature, that were implemented in the development of the artefact. These elements are: challenge, fantasy, curiosity, choice, rules, competition and goals.

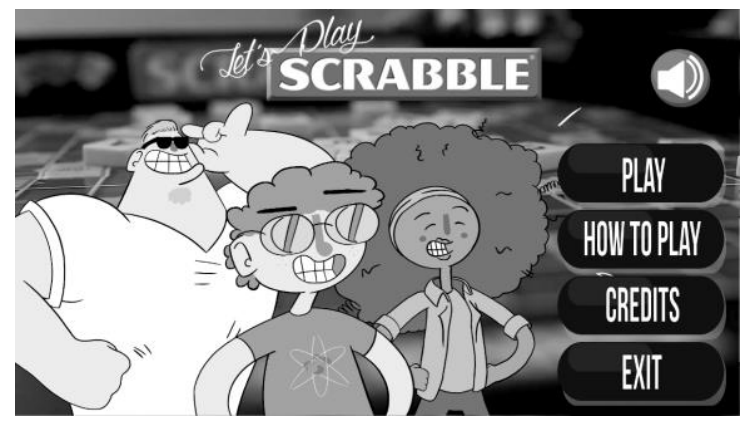

Figure 2. Main menu screen

The first screen that is visible to the user when the game is run, is the Main Menu screen (Figure 2). The main menu consists of a background image and five buttons. The background image depicts the three AI player characters and enforces the curiosity element of the artefact.

By showing the user these colourful depictions, their curiosity is piqued, contributing to the player's motivational level (Malone, 1981). Throughout the game, these character designs are used to pique and satisfy the curiosity of players, effectively raising their motivational level, leading to a more pleasant and memorable gaming experience.

The buttons on the main menu screen are simplistic, but clear, so as not to confuse the player, but rather to give clear and precise instructions. These buttons include: Play (starts a game), How to play (shows a short tutorial on the layout of the game, its elements and how it works), Credits (shows a list of all the individuals that contributed to the development process), Exit (leaves the game) and a button that can be used to toggle the sound (to ensure that users experience a pleasant time, with enough customizability to control the environment). Once the player clicks on the Play button, the game option screen will be displayed (Figure 3). Here the user 
has three customizable options to specify: Number of opponents, Difficulty, and Game mode. These options include the choice element, as it gives the players the choice to customize the game experience to suit their preferences.

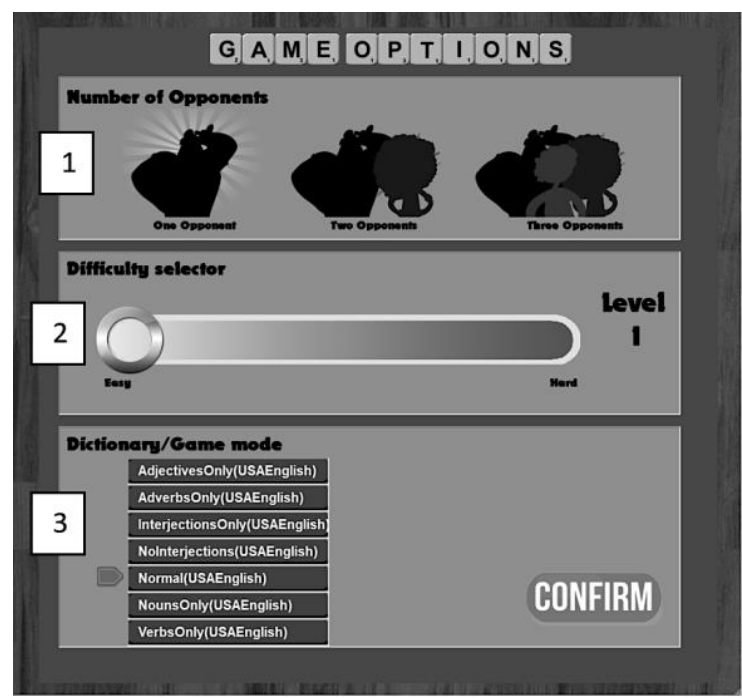

Figure 3. Game options screen

The difficulty selector is used to bring out the challenge element of the game. By picking a level that the player is comfortable with, a fair match can be created, ensuring that the player is not overwhelmed and preventing the player from always losing to the computer. It also ensures that the player is not underwhelmed, winning each game without being challenged.

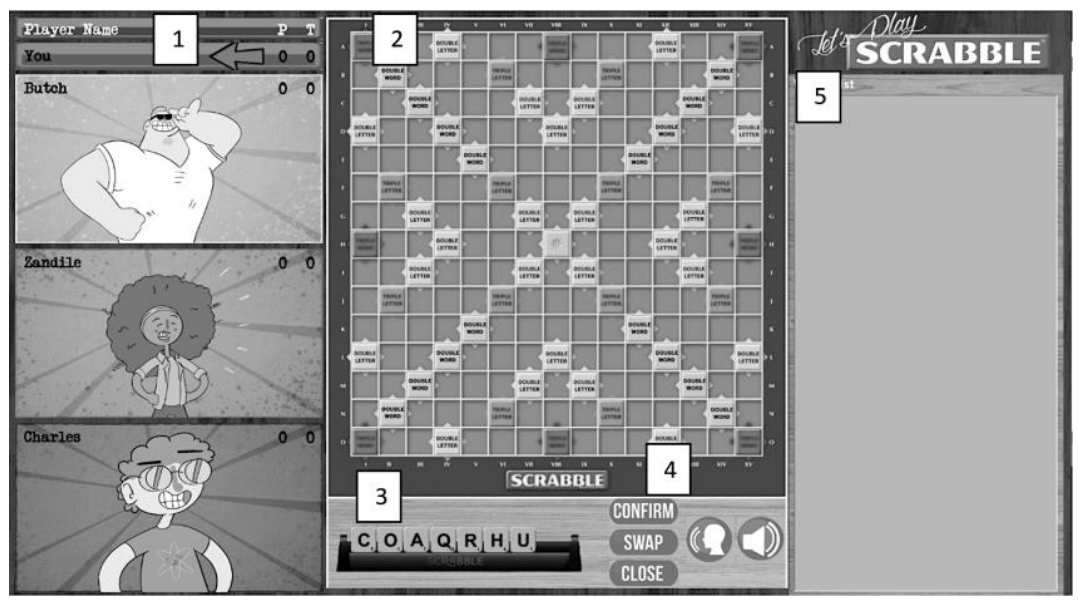

Figure 4. Game main screen

The game mode option is used to further enforce the educational element of the artefact. By setting up different game modes, the player can specify which words they want to play and thus which words they want to learn. Examples of these are game modes that only allow verbs or nouns or a game in another language.

The next screen shown is the main game screen (Figure 4). The screen is divided into three vertical parts, as to make it easier to look at without getting confused or overwhelmed.

On the left (1), the player's score and the opponent's scores are displayed, along with their character designs. The scores are displayed to remind the player of the competition and goal elements. By showing the current standings to the player, they will be reminded of their goal of winning and the opponents that they have to face, increasing motivation and experience (Charsky, 2010). The opponents also react to the current events, thus serving as an exogenous fantasy element that rewards the player with certain reactions and sounds, creating an immersive experience. 
The middle section is used as the playing space that consists of the board (2), the player rack and tiles (3) and the buttons (4). The board and tiles are used to enforce the rules element of the artefact, anchoring the game in reality and giving clear instructions of what is expected of the player. The tiles can be clicked and dragged onto the game board to add to the user's choice. The buttons are used to confirm the player's selection, swap tiles, close the current game session or mute the music or character sounds.

The final section of the main game screen is the word list. The word list is one of the most important educational elements of the artefact, as it does not only show the words that have been played during the game, but it also gives a precise definition for each word, enabling the player to learn these words while waiting for the AI players to complete their turns.

\section{SUMMARY AND CONCLUSION}

In this paper, a literature study was done about important factors regarding the given problem and the possible solutions. First, the history of educational games was discussed, giving the reader a better understanding of the genre and how it originated. This was followed by an explanation as to why educational games often fail and how effective it is when it succeeds. Next the elements necessary for optimal games were identified. Finally, a summary of Scrabble rules and gameplay was given, followed by strategies used to improve at the game. These strategies were used together with the heuristic algorithm to ensure that the AI program has a strategy when playing.

\section{REFERENCES}

Abelson, H. et al, 1974. LOGO Manual. Massachusetts Institute of Technology, Massachusetts, USA.

Almeida, L. C., 2012. The Effect of an Educational Computer Game for the Achievement of Factual and Simple Conceptual Knowledge Acquisition. Education Research International, Vol. 2012, Article ID 961279, 5 pages.

Amr, K. 2012. Learning through Games: Essential Features of an Educational Game. Syracuse University, USA.

Charsky, D.2010. From Edutainment to Serious Games: A Change in the Use of Game Characteristics. Games and Culture, Vol. 4, No. 2, pp 177-198.

Connolly, F. and Gren, D. 2012. Smart Scrabble playing - strategies and their impact. Kungliga Tekniska Högskolan, Stockholm, Sweden.

Hunicke, R. et al, 2004. MDA: A Formal Approach to Game Design and Game Research, Proceedings of the Challenges in Games AI Workshop, Nineteenth National Conference of Artificial Intelligence. San Jose, CA, USA, pp. 1-5.

Klopfer, E. et al, 2009. Moving learning games forward. The Education Arcade, Cambridge, MA, USA.

Kramer, W., 2015. What makes a game good? Game \& Puzzle design, Vol. 1, No 2, pp. 84-86.

Malone, T. W., 1981. Toward a Theory of Intrinsically Instruction. Cognitive Science, Vol. 5, No. 4, pp. 333-369.

Malone, T. W. and Lepper, M. R., 1987. Making learning fun: A taxonomy of intrinsic motivations for learning. Conative and Affective Process Analyses. Hillsdale, NJ, USA, Chapter 10.

Merino-Campos, C. and, del Castillo, H.: The benefits of active video games for educational and physical activity approaches: A systematic re- view. New Approaches in Educational Research, Vol. 5, No. 2, pp. 115-122.

Mildner, P. et al, 2015. From game characteristics to effective learning games: Evaluation of a component-based quiz game. Serious Games. JCSG 2015. Lecture Notes in Computer Science, vol. 9090, pp. 51-62. Springer International Publishing, Cham.

Needleman, A., 2017. A quick history of educational video games. Gamer Professionals. May 2017.

Stutt, T., 2010. Why educational games fail. Educational Technology and Change Journal. October 2010.

Virvou, M. et al. 2005. Combining software games with education: Evaluation of its educational effectiveness. Educational Technology \& Society. Vol. 8. No. 2, pp. 54-65.

Zin, N.A.M. and Yue, W.S. 2009. History educational games design. Proceedings of the 2009 International Conference on Electrical Engineering and Informatics, ICEEI 2009. vol. 01, pp. 269-275. 